\title{
On-demand sedation with propofol for colonoscopy-A prospective pilot study of the influence on short-term memory, psychomotor function and postural stability ${ }^{*}$
}

\author{
Mogens Rørbæk Madsen \\ Surgical Research Unit, Department of Surgery, Herning Regional Hospital, Herning, Denmark \\ Email: $\underline{\text { mogmad@rm.dk }}$
}

Received 30 July 2013; revised 30 August 2013; accepted 8 September 2013

Copyright (C) 2013 Mogens Rørbæk Madsen. This is an open access article distributed under the Creative Commons Attribution License, which permits unrestricted use, distribution, and reproduction in any medium, provided the original work is properly cited.

\begin{abstract}
Background: After sedated colonoscopy, patients are discharged on the basis of their subjective judgment that they have recovered, corroborated by the nursing staff. The aim of this study was to assess objectively whether patients were in fact fully recovered at the time of discharge, and to demonstrate whether the methods of testing applied could detect any influence of sedation on short-term memory, psychomotor function and postural stability. Methods: Twenty-two patients were investigated. At the beginning of the procedure, a defined bolus of propofol/alfentanil was given intravenously. During the procedure, an additional bolus was injected one or more times as requested by the patient. After colonoscopy, the patients stayed in the recovery room until the patients judged that they had recovered completely, which was also the judgement of the nursing staff at that time. Before colonoscopy and again before discharge, tests were performed of short-term memory, psychomotor function and postural stability (balance). Results: A positive correlation was found between the duration of colonoscopy and the amount of sedative given $(p<$ 0.03). No differences in short-term memory or postural control were found when measurements obtained before and after colonoscopy were compared. Reaction time was prolonged significantly after colonoscopy $(p<0.01)$, which was mainly due to prolongation of perception time $(p<\mathbf{0 . 0 0 3})$. No correlation was found between the observed reduction in psychomotor function and the amount of sedative given. Conclusions: The introduction of ultrashortacting sedative and hypnotic agents has facilitated out-patient colonoscopy. However, although they feel
\end{abstract}

*The author declares that there is no conflict of interest. that they have recovered fully, some patients are still affected by the sedative at the time of discharge, as demonstrated by tests of short-term memory, reaction time and postural stability.

Keywords: Colonoscopy; Sedation; Propofol; Reaction Time; Postural Stability

\section{INTRODUCTION}

After sedated colonoscopy, patients are usually discharged when they feel that they have recovered. Obviously, such a judgement is subjective and may be in error. The purpose of the present study was to measure by objective means whether the patients were in fact fully recovered at the time of discharge. Tests of short-term memory, reaction time and postural stability were performed to detect such possible impairment of recovery.

\section{PATIENTS AND METHODS}

Twenty-two patients (ASA I-II; 15 men and 7 women; median age 57 years, range 41 - 72 years) who underwent a scheduled colonoscopy to ensure the presence of a healthy colon after earlier removal of colorectal polyp(s) participated in the study.

Before colonoscopy, baseline testing was performed:

1. Short-term memory

WAIS test (Wechsler Adult Intelligence Scale-digit span) [1].

2. Psychomotor function (reaction time)

This was measured using Good Reaction equipment (Metitur, Jyväskylä; Figure 1) using visual stimuli. The two components of reaction time (perception time (msec) and movement time (msec)) were recorded separately (the method is described in [2]).

3. Postural stability (balance) 


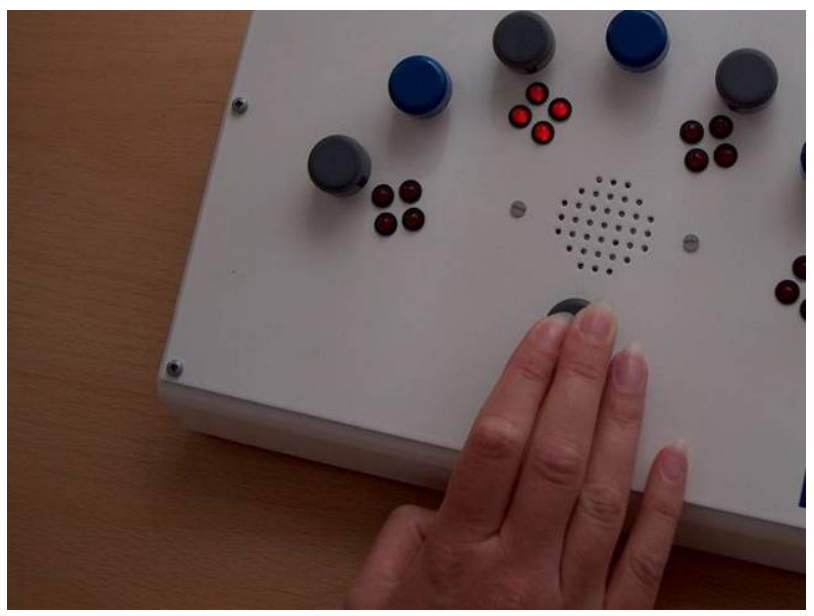

Figure 1. Testing of psychomotor reaction time.

This was measured using Good Balance equipment (Metitur, Jyväskylä; Figures 2 and 3). Postural sway, expressed as velocity moment $\left(\mathrm{mm}^{2} / \mathrm{sec}\right)$, was recorded with the eyes open and the eyes closed (the method is described in $[3,4])$.

Colonoscopy was carried out by one of three experienced endoscopists. At the beginning of the procedure, a bolus of $4 \mathrm{ml}$ of propofol/alfentanil $(9 \mathrm{mg} / \mathrm{ml}$ and 0.08 $\mathrm{mg} / \mathrm{ml}$, respectively) was given intravenously. During colonoscopy, a further bolus was injected one or more times as requested by the patient, and during the procedure, blood pressure, pulse, and oxygen saturation were monitored.

After colonoscopy, the patients were transferred to the recovery room and stayed there until they had recovered completely and were ready for discharge, as judged by the patients themselves and the nursing staff. The criteria included being fully awake, having no pain or nausea, and being capable of eating and drinking. At this time, all the tests described above were repeated.

Before the statistical analysis, a Spearman correlation test was carried out on the measurements obtained before and after colonoscopy to ensure effective pairing. The results obtained before and after colonoscopy were compared using the Wilcoxon signed-ranks test for matched pairs, with p-values $<0.05$ considered significant.

No particular ethical considerations were deemed to arise as a result of the methods of investigation and the patients entered the study anonymously after informed consent. The study was approved by the Danish Data Protection Agency, and conformed to the provisions of the Declaration of Helsinki.

\section{RESULTS}

Complete colonoscopy was performed in all patients. The median duration of investigation was 13 minutes (range 8 - 26 minutes). The patients received $14 \mathrm{ml}$ of

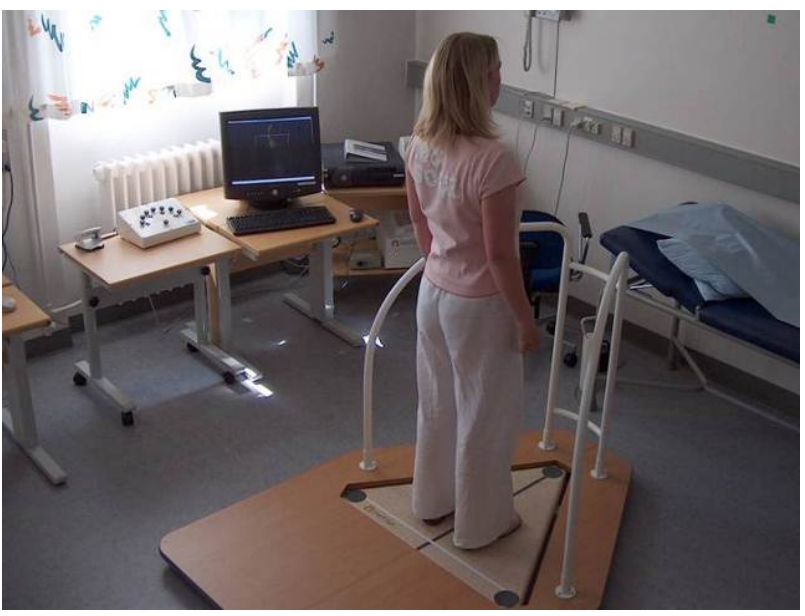

Figure 2. Testing of postural stability (balance).

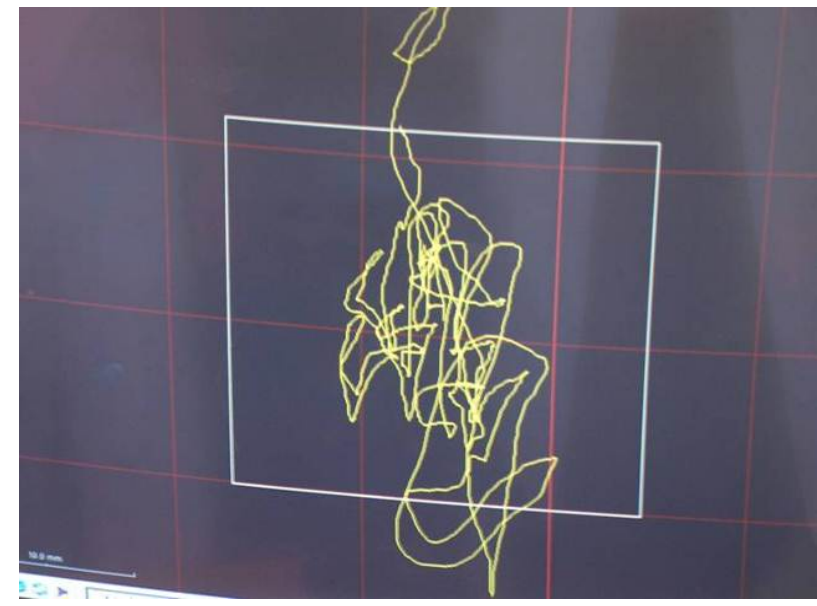

Figure 3. Monitor showing postural sway during testing.

sedative/analgesic (median; range 4 - $28 \mathrm{ml}$ ), which corresponds to a median of $126 \mathrm{mg}$ of propofol and $1.12 \mathrm{mg}$ of alfentanil. A linear correlation was demonstrated between the duration of colonoscopy and the amount of sedative $(\mathrm{ml} / \mathrm{kg})$ given $(\mathrm{r}=0.45, \mathrm{p}<0.03)$, but not between the amount of sedative given and the duration of stay in the recovery room, the median for which was 55 minutes (range 35 - 90 minutes).

\subsection{Short-Term Memory}

No statistically significant difference was found between the WAIS test score before colonoscopy (median score 9, range 7 - 12) and that after colonoscopy (median score 9, range 6 - 13).

\subsection{Psychomotor Function}

Overall reaction time was prolonged significantly after colonoscopy, from a median of $600 \mathrm{msec}$ to $680 \mathrm{msec}$ (p $<0.01$ ), with a maximum increase in one patient of 470 msec. The overall increase was due mainly to the pro- 
longation of perception time (from a median of $400 \mathrm{msec}$ to 460 msec, $\mathrm{p}<0.003$ ). Movement time did not increase significantly (from a median of $210 \mathrm{msec}$ to $230 \mathrm{msec}$ ). No correlation was found between the reduction in psychomotor function observed and the amount of sedative given.

\subsection{Postural Stability}

The velocity moment was increased significantly (i.e. balance was impaired) in measurements obtained with the eyes closed as compared with measurements with the eyes open, both before and after colonoscopy ( $\mathrm{p}<0.001$ ). In addition, the velocity moment was increased after colonoscopy as compared with before colonscopy, both with the eyes open (median: 6.35 and $6.45 \mathrm{~mm}^{2} / \mathrm{sec}$ ) and with the eyes closed (median: 8.8 and $8.9 \mathrm{~mm}^{2} / \mathrm{sec}$ ), but not to a statistically significant degree.

\section{DISCUSSION}

In some institutions, colonoscopy is performed routinely without sedation. However, the procedure may occasionally be painful and unpleasant, and in our experience most patients prefer some type of sedation together with analgesia. Until a few years ago, diazepam and pethidine were used widely for this purpose, but in recent years newer, short-acting agents, such as propofol and alfentanil, have been used increasingly. The use of these agents has been shown to be safe in several studies [5-11], and has been reported in a Cochrane review [12]. It is a matter of discussion in the literature whether propofol should be used alone or in combination with opoids and/or benzodiazepines. Whether propofol should always be administered by anaesthesiologists is also controversial. However, the present study did not aim to address these issues.

The advantage of using propofol lies in its rapid onset and short duration of effect. The mean recovery time after propofol-sedated colonoscopy has been reported to range from 5 to 45 minutes [5-9].

In the present study, the patients had recovered clinically, as judged by themselves and the recovery room staff, after a median of 55 minutes. However, by objective measurement, they had not recovered fully, because the median reaction time at this point was still impaired significantly when compared with pre-procedural, baseline values. The reduced reaction time was not correlated with the amount of propofol administered, which may suggest a degree of individual variation in the metabolism and elimination of propofol. It also probably reflects the biphasic elimination of propofol, of which the secondary phase is known to last from 3 to 6 hours. Propofol is distributed mainly in brain tissue, so it exerts its effects primarily on brain function. This was demon- strated in the present study by the fact that the impairment in the overall reaction time was due mainly to reduced perception time and not to decreased motor function.

It is well known that sedation with benzodiazepines or barbiturates impairs cognitive and psychomotor performance, but to our knowledge only one study [6] has addressed these issues in detail in relation to sedation with propofol. Theodorou et al. [6] found a reduced reaction time after 30 minutes, but not after 60 minutes. This difference in comparison to our study is probably explained by the fact that the patients in our study received larger doses of propofol (median: $126 \mathrm{mg}$ ). Findings for the mean dose of propofol necessary for the completion of colonoscopy vary among different studies, from 66 to $141 \mathrm{mg}[5,7,8,13]$.

As assessed by the WAIS test, we did not find any influence on short-term memory. This contrasts with the findings of Theodorou et al. [6], who found a decrease in short-term memory (using the Picture Recall test) up to 2 hours after colonoscopy. In addition, Theodorou et al. found impairment of other cognitive functions (by use of the Digit Symbol Substitution test, Grooved Pegboard test and Speed of Comprehension test), which lasted for 2 hours after colonoscopy. However, in the latter study, patients were given midazolam in addition to propofol and fentanyl, which may explain these findings. Similarly, Padmanabhan et al. [14] found cognitive impairment after sedation with propofol in combination with midazolam and/or fentanyl by using the CogState test. It is unclear which test of cognitive function is the more appropriate to use when this combination of drugs is administered.

Postural stability (balance) is maintained as the result of an unconscious, complex, but synchronized interaction between the central and peripheral nervous systems and several individual muscles and groups of muscles. In the upright position, the body will constantly sway in all directions, and the magnitude of sway and the speed with which it is corrected are an expression of the ability to maintain postural balance. This ability, which can be measured as described previously [3,4], is affected negatively by many diseases and neuromuscular conditions and also by medication. However, the effect of propofol or other anaesthetics on postural balance has not been tested previously. As expected, postural control was found to be poorer with the eyes closed than with the eyes open, but only a statistically non-significant further impairment could be demonstrated after sedated colonoscopy. Further studies are needed to determine whether our finding applies in all cases or was due to the limited size of the test population.

In conclusion, in spite of the use of propofol, a shortacting anaesthetic agent, some patients were still affected 
by sedation at the time of discharge after colonoscopy, although they judged themselves to have recovered fully and their judgement was corroborated by nursing staff. Sedation results in impaired psychomotor function, and patients should be warned about the fact that they may not have recovered fully at the time of discharge, because it might have serious consequences if they perform certain activities, for example, driving a motor vehicle or operating machinery.

This pilot study also shows that the assessment of reaction time (with separate recording of perception time and movement time) and the measurement of postural control could be useful tools in future prospective comparative studies of the effects of sedation.

\section{REFERENCES}

[1] L. I. Iverson and D. S. Tulsky, "Detecting Malingering on the WAIS-III Unususal Digit Span Performance Patterns in the Normal Population and in Clinical Groups," Archives of Clinical Neuropsychology, Vol. 18, No. 1, 2003, pp. 1-9.

[2] P. Era, J. Jokela and E. Heikkinen, "Reaction and Movement Times in Men of Different Ages: A Population Study," Perceptual and Motor Skills, Vol. 63, No. 1, 1986, pp. 111-130. doi:10.2466/pms.1986.63.1.111

[3] P. Era and E. Heikkinen, "Postural Sway during Standing and Unexpected Disturbance of Balance in Random Samples of Men of Different Ages," Journal of Gerontology, Vol. 40, No. 3, 1985, pp. 287-295. doi:10.1093/geronj/40.3.287

[4] P. Era, M. Schroll, H. Ytting, I. Gause-Nilsson et al., "Postural Balance and Its Sensory-Motor Correlates in 75-Years-Old Men and Women: A Cross-National Comparative Study,” Journal of Gerontology, Vol. 51, No. 2, 1996, pp. 53-63.

[5] D. Külling, A. C. Fantin, P. Biro et al., "Safer Colonoscopy with Patient-Controlled Analgesia and Sedation with Propofol and Alfentanil," Gastrointestinal Endoscopy, Vol. 54, No. 1, 2001, pp. 1-7. doi:10.1067/mge.2001.116174

[6] T. Theodorou, P. Hales, P. Gillespie et al., "Total Intraveneous versus Inhalation Anaesthesia for Colonoscopy:
A Prospective Study of Clinical Recovery and Psychomotor Function,” Anaesth Intensive Care, Vol. 1, No. 2, 2001, pp. 124-136.

[7] L. B. Cohen, A. N. Dubovsky, J. Aisenberg et al., "Propofol for Endoscopic Sedation: A Protocol for Safe and Effective Administration by the Gastroenterologist," Gastrointestinal Endoscopy, Vol. 58, No. 5, 2003, pp. 725732. doi:10.1016/S0016-5107(03)02010-8

[8] E. Bright, C. Roseveare, D. Dalgleish et al., "PatientControlled Sedation for Colonoscopy: A Randomized Trial Comparing Patient-Controlled Administration of Propofol and Alfentanil with Physician-Administered Midazolam and Pethidine,” Endoscopy, Vol. 35, No. 8, 2003, pp. 683-687. doi:10.1055/s-2003-41519

[9] R. Rudner, P. Jalowiecki, P. Kawecki et al., "Conscious Analgesia/Sedation with Remifentanil and Propofol versus Total Intravenous Anesthesia with Fentanyl, Midazolam, and Propofol for Outpatient Colonoscopy," Gastrointestinal Endoscopy, Vol. 57, No. 6, 2003, pp. 657-663. doi:10.1067/mge.2003.207

[10] M. E. VanNatta and D. K. Rex, "Propofol Alone Titrated to Deep Sedation versus Propofol in Combination with Opoids and/or Benzodiazepines and Titrated to Moderate Sedation for Colonoscopy," The American Journal of Gastroenterology, Vol. 101, No. 10, 2006, pp. 2209-2217. doi:10.1111/j.1572-0241.2006.00760.x

[11] D. K. Rex, V. P. Deenadayalu, E. Eid et al., "EndoscopistDirected Administration of Profofol: A Worldwide Safely Experience,” Gastroenterology, Vol. 137, No. 4, 2009, pp. 1229-1237. doi:10.1053/j.gastro.2009.06.042

[12] H. Singh, W. Poluha, M. Cheung et al., "Propofol for Sedation during Colonoscopy," Cochrane Database of Systematic Reviews, Vol. 8, No. 4, 2008, Article ID: CD006268.

[13] L. T. Heuss, J. Drewe, P. Schnieper et al., "Patient-Controlled versus Nurse-Administered Sedation with Propofol during Colonoscopy. A Prospective Randomized Trial," The American Journal of Gastroenterology, Vol. 99, No. 3, 2004, pp. 511-518. doi:10.1111/j.1572-0241.2004.04088.X

[14] U. Padmanabhan, K. Leslie, A. S. Y. Eer et al., "Early Cognitive Impairment after Sedation for Colonoscopy: The Effect of Adding Midazolam and/or Fentanyl to Propofol,” Anesthesia \& Analgesia, Vol. 109, No. 5, 2009, pp. 1448-1455. 